This item was submitted to Loughborough's Research Repository by the author.

Items in Figshare are protected by copyright, with all rights reserved, unless otherwise indicated.

\title{
A parallel approach to row-based VLSI layout using stochastic hill-climbing
}

PLEASE CITE THE PUBLISHED VERSION

PUBLISHER

(C) Springer

LICENCE

CC BY-NC-ND 4.0

\section{REPOSITORY RECORD}

Newton, Matthew, Ondrej Sykora, Mark S. Withall, and Imrich Vrt'o. 2019. "A Parallel Approach to Row-based VLSI Layout Using Stochastic Hill-climbing”. figshare. https://hdl.handle.net/2134/3917. 
This item was submitted to Loughborough's Institutional Repository (https://dspace.lboro.ac.uk/) by the author and is made available under the following Creative Commons Licence conditions.

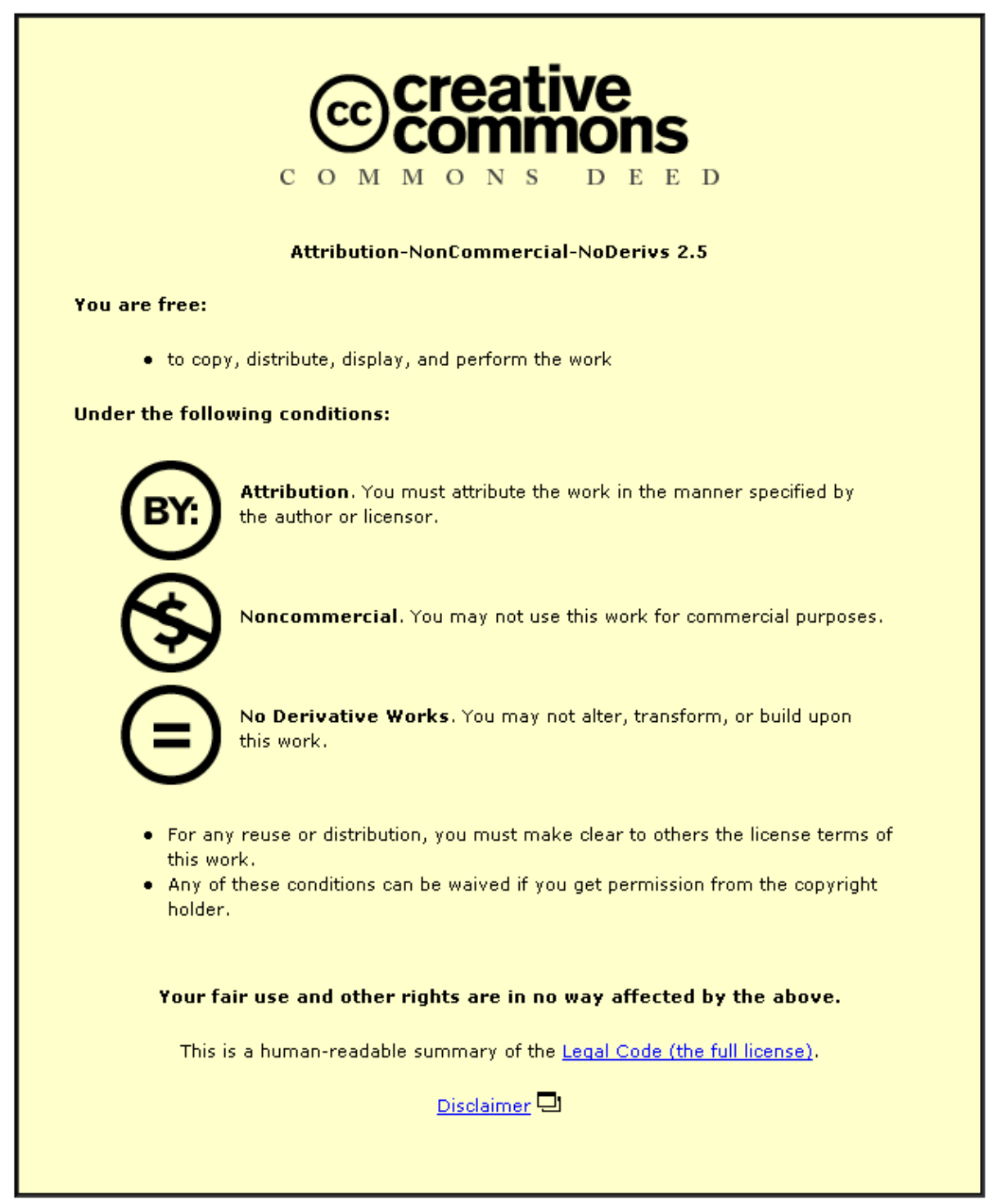

For the full text of this licence, please go to: http://creativecommons.org/licenses/by-nc-nd/2.5/ 


\title{
A Parallel Approach to Row-Based VLSI Layout using Stochastic Hill-Climbing ${ }^{\star}$
}

\author{
Matthew Newton ${ }^{1}$, Ondrej Sýkora ${ }^{1}$, Mark Withall ${ }^{1}$, and Imrich Vrt'o ${ }^{2}$ \\ 1 Department of Computer Science, Loughborough University, Loughborough, Leics. \\ LE11 3TU, United Kingdom \\ \{m.c.newton, o.sykora,m.s.withall2\}@lboro.ac.uk \\ http://parc.lboro.ac.uk/ \\ 2 Department of Informatics, Institute of Mathematics, Slovak Academy of Sciences, \\ P.O.Box 56, 84000 Bratislava, Slovak Republic \\ imrich@ifi.savba.sk
}

\begin{abstract}
Parallel algorithms based on stochastic hill-climbing and parallel algorithms based on simple elements of a genetic algorithm for the one-sided bipartite crossing number problem, used in row-based VLSI layout, were investigated. These algorithms were run on a PVM cluster. The experiments show that the parallel approach does not bring faster computation but it does, however, much more importantly, bring a better quality solution to the problem, i.e. it generates drawings with lower numbers of pairwise edge crossings.
\end{abstract}

Keywords. Genetic Algorithms, Distributed Problem Solving

\section{Introduction}

Graph drawing addresses the problem of finding a layout of a graph that satisfies given aesthetic and readability objectives. One of the basic problems with the drawing of bipartite graphs is that of two layer automatic drawing where two vertex partitions are put in distinct points on two parallel lines and edges are drawn as straight line segments. This type of drawing is the basic building block used for drawing hierarchical graphs[4,10] or producing row-based VLSI layouts[12]. Probably the most important objective is minimisation of the number of edge crossings in the drawing, as the aesthetic and readability of graph drawings depend on the number of edge crossings (see [11]), and VLSI layouts containing less crossings are more easily realisable and consequently cheaper. There are two basic variants of the problem: the one-sided and two-sided crossing minimisation problems. In the one-sided problem the vertices of one part of the bipartite graph are placed in fixed positions on one line (known as the fixed side) and the positions of the vertices of the other part, on the other line (known as the free side), are found so that the number of pairwise edge crossings is minimised.

\footnotetext{
* Research of the first two and last authors was supported by the EPSRC grant GR/R37395/01. Research of the second and the last authors was supported by the Slovak Scientific Grant Agency grant No. 2/7007/20.
} 
As both problems are NP-hard[5,7], a lot of different methods-heuristics and approximation algorithms - to solve the problem have been designed (see e.g. [1, $3])$.

Parallel computing has been a valuable tool for improving running time and enlarging feasible sizes of problems and it has become an important economic and strategic issue. This paper focuses on the parallel implementation of genetic algorithms and stochastic hill-climbing for one-sided bipartite graph drawing. In comparison with the best sequential one-sided methods penalty minimization and sifting (see [3]), the presented heuristics are slower but they produce much higher quality results. The experiments show that better results are achieved than penalty minimization and sifting with the sequential version of the algorithm but using parallel (cluster) computing makes it possible to improve the quality of the solution in a significant way.

\section{Notation}

Let $G=(V, E), V=V_{0} \cup V_{1}$ be a bipartite graph with vertex partitions $V_{0}$ and $V_{1}$. A bipartite drawing of $G$ is obtained by placing the vertices of $V_{0}$ and $V_{1}$ into distinct points on two horizontal lines, $y_{0}, y_{1}$ respectively, and drawing each edge with one straight line segment. It is assumed that $y_{0}$ is the line $y=0$ and $y_{1}$ is the line $y=1$.

Any bipartite drawing of $G$ is identified by two permutations $\pi_{0}$ and $\pi_{1}$ of the vertices on $y_{0}$ and $y_{1}$ respectively. It is assumed that the permutation $\pi_{1}$ is fixed. The problem of the one-sided bipartite drawing of $G$ is the problem of finding a permutation $\pi_{0}$ that minimises the number of pairwise edge crossings in the corresponding bipartite drawing.

Let $\operatorname{bcr}\left(G, \pi_{0}, \pi_{1}\right)$ denote the total number of crossings in the bipartite drawing represented by the permutations $\pi_{0}$ and $\pi_{1}$. The bipartite crossing number of $G$ related to the fixed permutation $\pi_{1}$, denoted by $\operatorname{bcr}\left(G, \pi_{1}\right)$, is the minimum number of crossings over all $\pi_{0}$. Clearly, $b c r\left(G, \pi_{1}\right)=\min _{\pi_{0}} b c r\left(G, \pi_{0}, \pi_{1}\right)$.

\section{Stochastic Hill-Climbing for Bipartite Crossings}

The space of orderings of the free side for the one-sided bipartite drawing problem can be represented by all permutations of $\{0,1,2, \ldots, n-1\}$, where $n$ is the number of vertices on the free side. To find a solution of the one-sided bipartite drawing problem means to search this space for a permutation providing the minimum number of crossings. To move across the space it is possible to use some steps (local or non-local). The new visited permutation can be evaluated and then accepted if it fulfils a condition (e.g. it has a lower or equal number of crossings). Searching is then continued from the new accepted permutation. If the new permutation was not accepted, the old permutation is used. A natural local step is vertex swapping which actually corresponds to transposition in permutations. The space of permutations with vertex swapping used for generating a new candidate for a solution can be represented by the so called complete 
transposition graph $C T_{n}=(V, E)$. The graph has $n$ ! nodes labelled by all distinct permutations of the numbers from $\{0,1,2, \ldots, n-1\}$ and two vertices of $C T_{n}$ are adjacent if the corresponding permutations can be obtained from each other by a transposition of two elements. The transposition graph belongs to the class of Caley graphs[6], and its diameter is $n-1$. To search the space for a solution, a genetic algorithm approach was used in [8]. The typical structure of a genetic algorithm is shown in Algorithm 1.

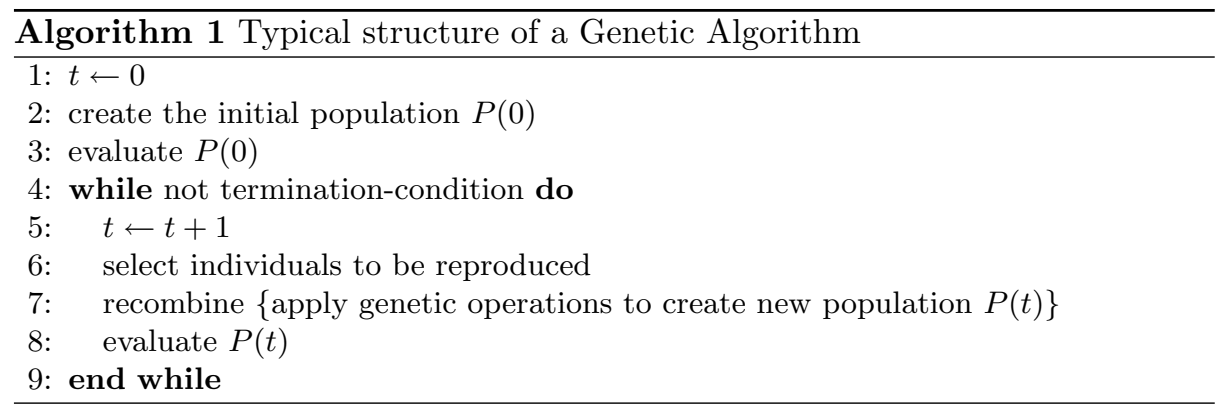

To create a concrete genetic algorithm it is necessary to fix some of the parameters of the above general scheme. It is necessary to define crossover (usually a binary operation) and mutation, and the way that the candidates for parents and mutation are chosen. Then the initial population should be chosen. In general, genetic algorithms provide better results than other, simpler, heuristics, the main problem being long computation time. To avoid it, it is possible to simplify the procedure by, for example, removing crossover, simplifying mutation and using a simple evaluation function. Very good results were achieved, better than the best heuristics in this area - sifting[9] and penalty minimization[3] - by mutation reduced to swapping two randomly chosen vertices and by applying hill-climbing using a differential fitness function (only the changed edges were checked), see Algorithm 2. This approach has not been applied before.

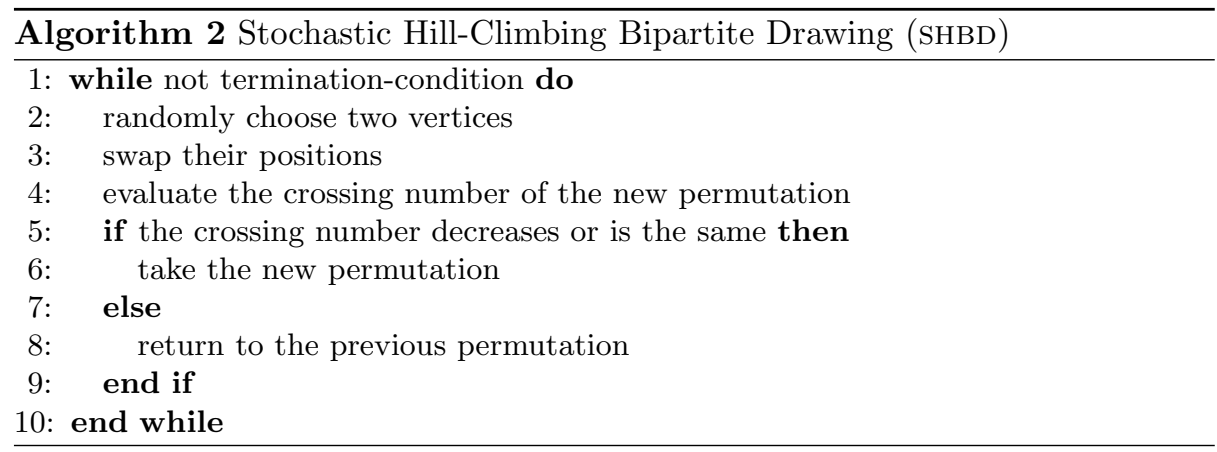


The termination condition can be defined by the number of iterations, the number of iterations that have passed since the last swap that gave an improvement to the crossing number (the number of stagnations) or a required crossing number can be given for the algorithm to achieve. From experimental observation, a run time of $O(f(n))$, where $f(n)$ appears to be at most a cubic function of the number of vertices, seems to be a good approximation.

\section{Parallel Algorithms}

Parallel computing has been a valuable tool for improving running time and enlarging feasible sizes of problems and it has become a key economic and strategic issue. Strong efforts are put into developing standards for parallel programming environments, such as PVM (Parallel Virtual Machine), MPI (Message Passing Interface), BSP (Bulk Synchronous Parallel) and HARNess. Among these, one of the most common is currently PVM (see http://www.csm.ornl.gov/pvm/), although the situation is changing rapidly (see for example [2]). Algorithm 3 shows the general scheme of the parallel genetic algorithm used.

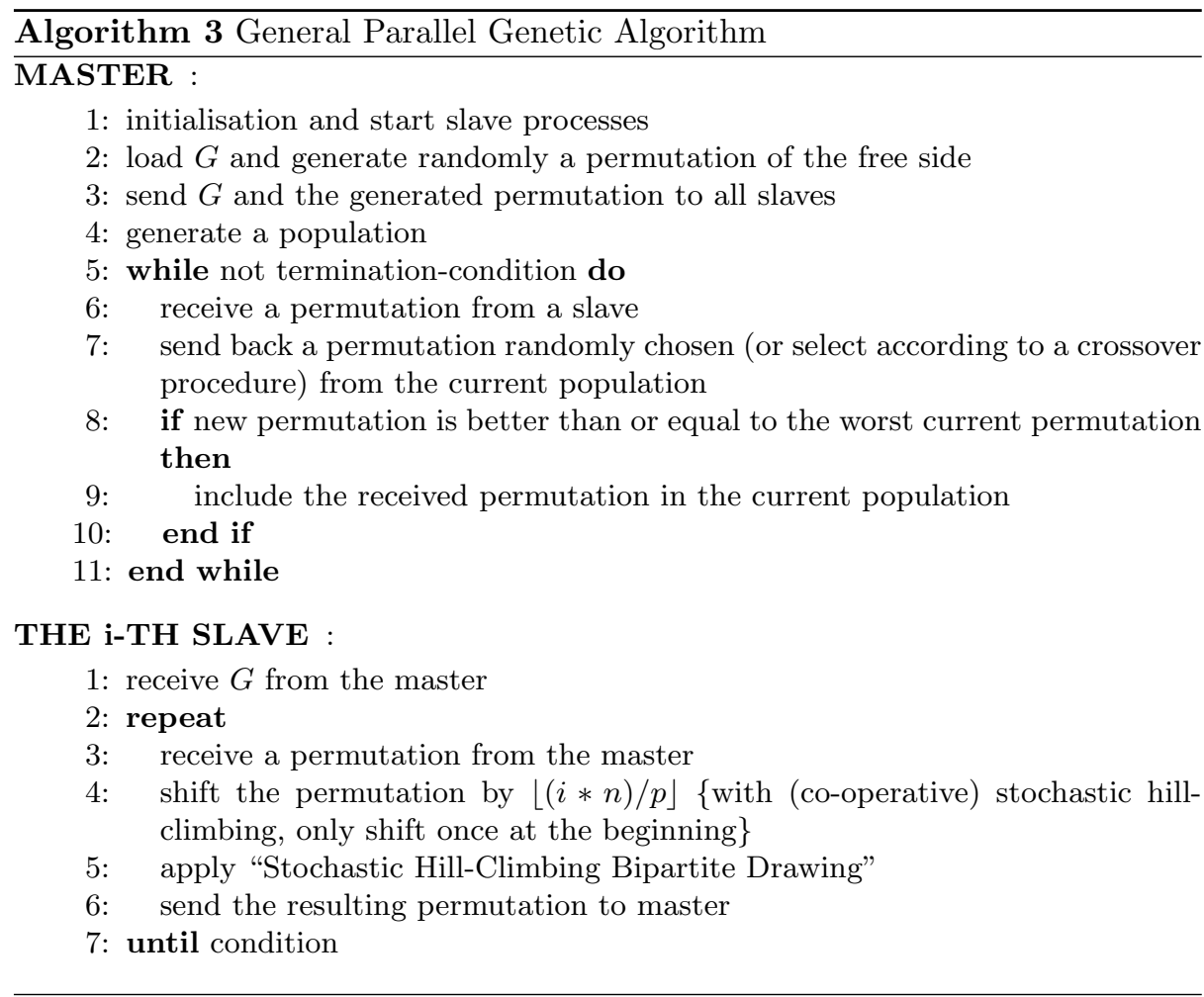


In the above approach, crossover, if any, is realised in the master. The mutation operator can be applied in parallel on every new permutation, or when stagnation has occurred. A parallel stochastic hill-climbing approach was used where the master randomly generates permutations that are sent to the slaves. A mutation of acyclic shift by $\lfloor i n / p\rfloor$ positions is applied there. The mutations will generate permutations that are at least at distance $\lfloor n / 2\rfloor$ from each other because a permutation in the worst case can contain at most $\lfloor n / 2\rfloor$ cycles. This means that the mutations ensure a good distribution of starting positions over the space of permutations (which is probably the reason why the algorithms produce such good results). A slave finishes processing after some predefined number of runs of the procedure SHBD or after some predefined number of stagnations (where there has been no change of the best permutation). As soon as the slave halts, it sends the resulting permutation to the master and the master chooses the best one from all permutations sent by the slaves and halts.

The approach gave better results than sequential SHBD in a shorter time (see Figures 1,2 and 3), which is not too surprising. The method of parallel SHBD was improved by introducing co-operation of slaves, by means of the master working as follows: the master resends a new permutation to the slave from which it has just received a message. It can either be the best or randomly chosen from those maintained as a current population, or it may be created by a crossover operation. In the experiments a crossover operation which would make improvements to the current population could not be found, so it was decided not to use one.

Mutation was used to further improve the co-operative parallel stochastic hillclimbing. This method took much more time but improvements to the crossing number over the previous parallel SHBD were observed.

\section{Experimental Procedure}

The parallel algorithms were implemented in C, running on 46 Sun ULTRAsparc 5 workstations running Solaris 7 . These were connected with $100 \mathrm{Mb} / \mathrm{s}$ Ethernet via 3Com SuperStack II baseline 10/100 24 port switches. The algorithms were compiled with GNU C compiler version 2.95.2. PVM was used for the parallel communication and was configured as five parallel computers, each with 9 processors; one master and eight slaves. Another computer ran a job control system that was written in Perl to keep experiments running on the five machines.

The parallel and sequential algorithms, graph generators and the graphs used in the experiments are available from http://parc.lboro.ac.uk/.

\subsection{Graph Test Sets}

Two main types of graph where used in the experiment. Specific classes of graph, such as meshes, cycles, complete binary trees and caterpillars, were used to test the algorithm so that specific target crossing numbers were available. Randomly 
generated graphs were used as the main test to find out how the algorithm performs for more general types of graph.

Standard Graphs Several standard graphs were used as benchmarks in the experiments. These graphs were generated so that the fixed side in the drawing was in its optimal ordering, while the free side permutation was generated randomly. The time for the algorithm to reach the known crossing number was measured. Complete binary trees (an $n$-level complete binary tree has a bipartite crossing number $\left(\frac{n}{3}-\frac{11}{9}\right) 2^{n}+\frac{2}{9}(-1)^{n}+2$, see [13]), cycles (with a bipartite crossing number $\frac{n}{2}-1$, if there are $n$ vertices in the cycle) and caterpillars (without crossings in the optimal drawing) have been used to test if the algorithms are working correctly. Rectangular meshes, $3 \times n$, (with a bipartite crossing number equal to $5 n-6$, see [14]) have been used as a more challenging problem than cycles.

Random Graphs The random graphs used were generated based on edge density. The generator took two arguments, $n$ (the number of vertices) and $p \in[0 . .100]$ (the percentage chance that an edge will exist between two vertices). The general graphs were generated with sizes $n \in[100,200,300, \ldots, 1000]$ and densities of $10 \%, 1 \%$ and $0.1 \%$. The minimum crossing number was not known so the fixed side could not be placed optimally.

\subsection{Test Runs}

The standard graphs were generated with sizes $n \in[100,200,300, \ldots, 1000]$ and were run on the algorithms to find out how well they performed against a known solution. It was possible to see that the parallel stochastic hill-climbing method was slightly faster in reaching the known crossing number than the co-operative stochastic hill-climbing algorithm.

Each one of the generated random graphs was initially run on the parallel stochastic hill-climbing algorithm. As the exact crossing number was not known, for each of the graphs, they were run for 3 minutes each and then stopped. The crossing number that they had then reached and the time taken to reach that number were recorded. Each of the algorithms was run 10 times and the average time and crossing number were calculated.

The graphs were then run on the parallel system running the co-operative stochastic hill-climbing algorithm, first without mutation and then with. A sequential hill-climbing version was also run for each of the machines. All experiments were, again, run 10 times each. The graphs in Figures 1, 2 and 3 show the comparisons between the four results for random graphs where $n=500$. The results from the sequential stochastic hill-climbing algorithm have been shown in full due to the large diversity of the data. 


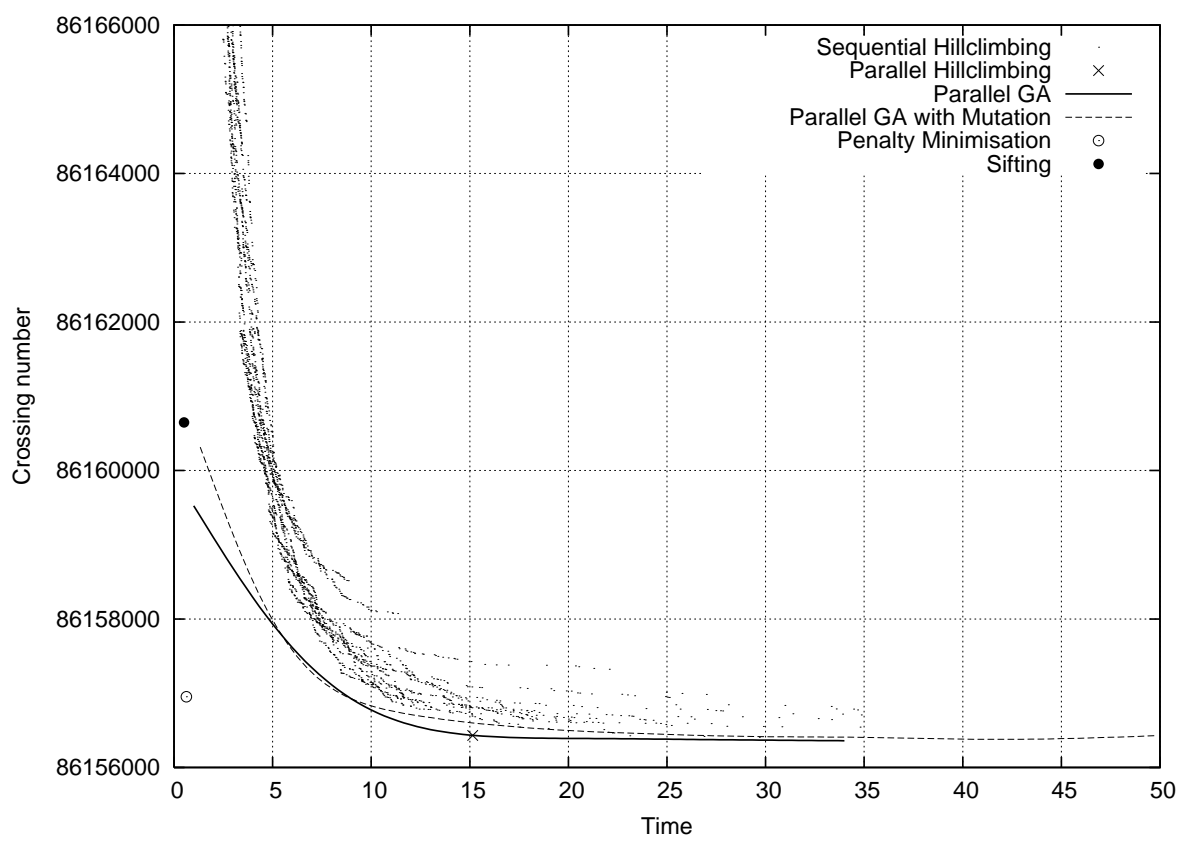

Fig. 1. Random graph, $n=500, p=10 \%$

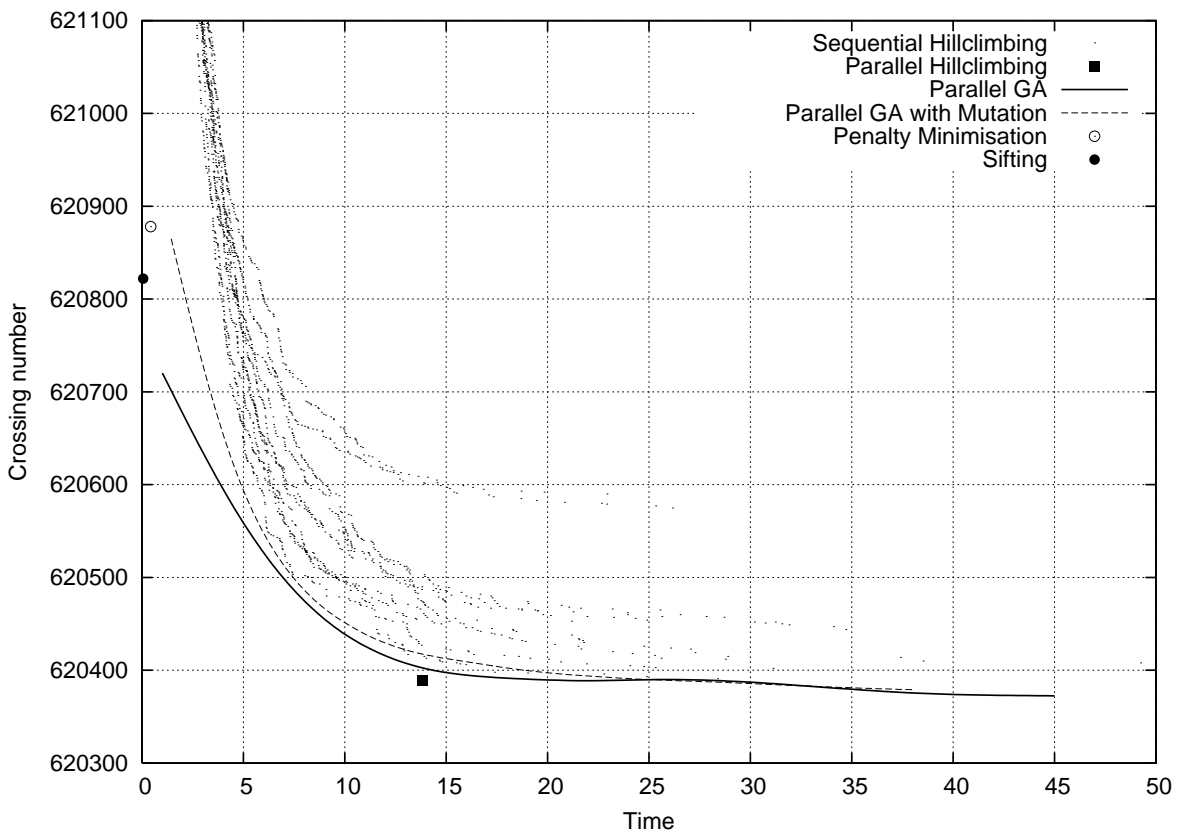

Fig. 2. Random graph, $n=500, p=1 \%$ 


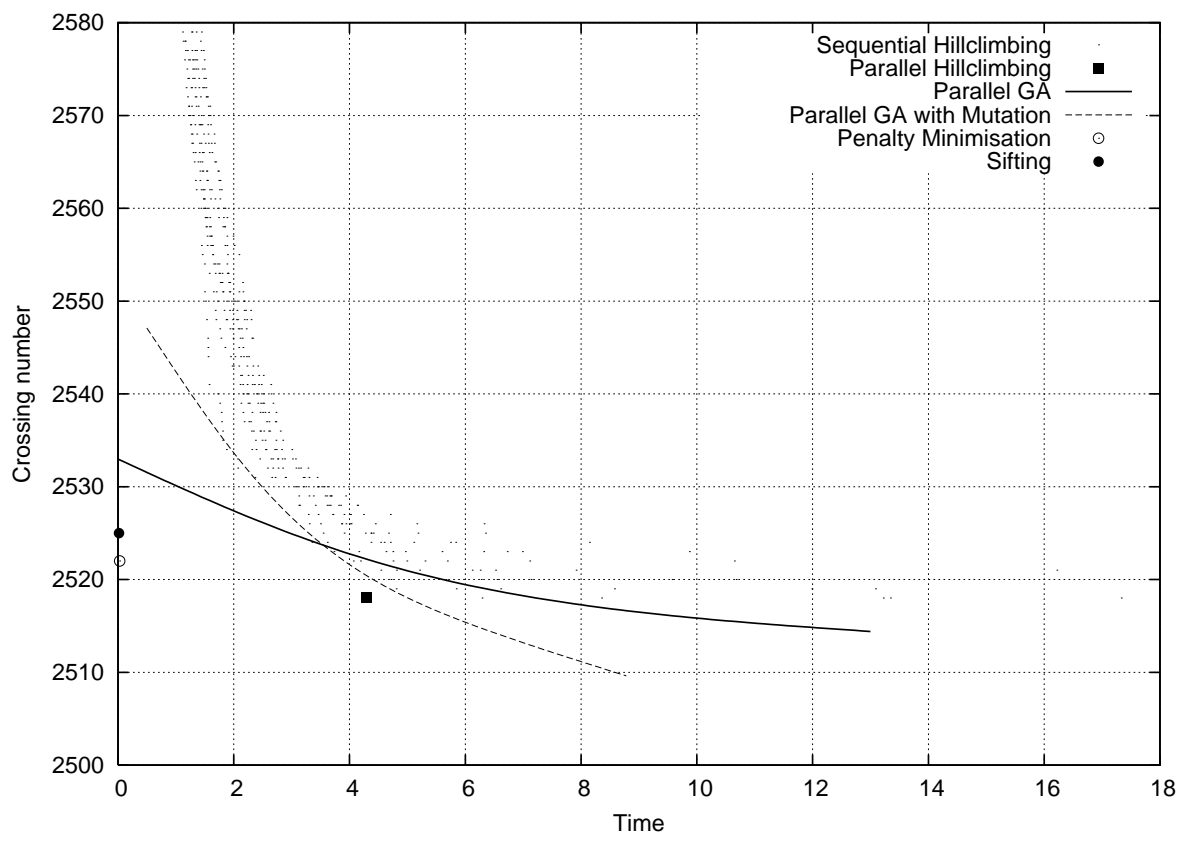

Fig. 3. Random graph, $n=500, p=0.1 \%$

\section{Summary and Conclusions}

The experiments were run for several different classes of graph using parallel stochastic hill-climbing and parallel genetic algorithms. For the results it follows that the parallel SHBD algorithms can be used to find better solutions to the onesided bipartite drawing problem, used in row-based VLSI layout, than the best available sequential heuristics such as penalty minimization and sifting. In addition, the SHBD can run much faster than a standard black-box algorithm due to the ability to implement a differential fitness evaluation. If co-operative stochastic hill-climbing is used, especially with mutation, the crossing number can be further improved (as compared to the results of the other parallel algorithms) at the expense of time.

\section{References}

1. O. Bastert, C. Matuszewski. (2001). Layered drawings of digraphs, In: Drawing Graphs, Methods and Models (eds. M. Kaufmann, D. Wagner), Lecture Notes in Computer Science 2025, Springer Verlag, 87-118.

2. J. Dongarra, G.A. Geist, J.A. Kohl, P.M. Papadopoulos, S. Scott, V. Sunderam. HARNESS: Heterogeneous Adaptable Reconfigurable Networked Systems. http:// www . csm. ornl . gov/harness/. 
3. C. Demetrescu., I. Finocchi. (2001). Removing cycles for minimizing crossings. J. Experimental Algorithmics. To appear.

4. G. Di Battista, P. Eades, R. Tamassia, I.G. Tollis. (1999). Graph Drawing: Algorithms for Visualization of Graphs. Prentice Hall.

5. P. Eades, N. Wormald. (1994). Edge crossings in drawings of bipartite graphs, Algorithmica, 11 379-403.

6. J.A. Gallian. (1989). A survey: recent results, conjectures and open problems in labelling graphs, J. Graph Theory 13 491-504.

7. M.R. Garey, D.S. Johnson. (1983). Crossing number is NP-complete, SIAM J. Algebraic Discrete Meth., 4 312-316.

8. E. Mäkinen, M. Sieranta. (1994). Genetic algorithms for drawing bipartite graphs, International J. Computer Mathematics 53 157-166.

9. C. Matuszewski, R. Schönfeld, P. Molitor. (1999). Using sifting for $k$-layer straightline crossing minimization, in: Proc. 7th International Symposium on Graph Drawing (GD'99), LNCS 1731, 217-224.

10. P. Mutzel. (2001). Optimization in leveled graphs. In: M. Pardalos, C.A. Floudas (eds.): Encyclopedia of Optimization. Kluwer, Dordrecht.

11. H. Purchase. (1998). Which aesthetic has the greatest effect on human understanding?, in Proc. Symposium on Graph Drawing, GD'97, Lecture Notes in Comput. Sci., 1353, Springer Verlag, Berlin, 248-261.

12. M. Sarrafzadeh, C.K. Wong. (1996). An Introduction to VLSI Physical Design, McGraw Hill, New York, NY.

13. F. Shahrokhi, O. Sýkora, L.A. Székely, I. Vrťo. (1998). On bipartite crossings, largest biplanar subgraphs, and the linear arrangement problem, in Proc. Workshop on Algorithms and Data Structures, WADS'97, Lecture Notes in Comput. Sci. 1272, Springer-Verlag, Berlin, 55-68.

14. F. Shahrokhi, O. Sýkora, L.A. Székely, I. Vrťo. (2000). A new lower bound for the bipartite crossing number with algorithmic applications, Theoretical Computer Science 245 281-294. 